\title{
Glycoprotein biosynthesis in Chlamydomonas
}

\author{
A mannolipid intermediate with the properties of a short-chain $\alpha$-saturated polyprenyl monophosphate
}

\author{
Winfried C. LANG \\ Fachbereich Biologie der Universität Kaiserslautern, Postfach 3049, D-6750 Kaiserslautern, Federal Republic \\ of Germany
}

(Received 1 February 1984/Accepted 21 February 1984)

\begin{abstract}
A crude membrane preparation of the unicellular green alga Chlamydomonas reinhardii was found to catalyse the incorporation of $\mathrm{D}-\left[{ }^{14} \mathrm{C}\right]$ mannose from GDP-D- $\left[{ }^{14} \mathrm{C}\right]-$ mannose into a chloroform/methanol-soluble compound and into a trichloroacetic acid-insoluble polymer fraction. The labelled lipid revealed the chemical and chromatographic properties of a short-chain (about $\mathrm{C}_{55}-\mathrm{C}_{65}$ ) $\alpha$-saturated polyprenyl mannosyl monophosphate. In the presence of detergent both long-chain $\left(\mathrm{C}_{85}-\mathrm{C}_{105}\right)$ dolichol phosphate and $\alpha$-unsaturated undecaprenyl phosphate $\left(C_{55}\right)$ were found to be effective as exogenous acceptors of D-mannose from GDP-D- $\left[{ }^{14} \mathrm{C}\right]$ mannose to yield their corresponding labelled polyprenyl mannosyl phosphates. Exogenous dolichyl phosphate stimulated the incorporation of mannose from GDP-D- $\left[{ }^{14} \mathrm{C}\right]$ mannose into the polymer fraction 5-7-fold, whereas the mannose moiety from undecaprenyl mannosyl phosphate was not further transferred. Authentic dolichyl phosphate $\left[{ }^{3} \mathrm{H}\right]-$ mannose and partially purified mannolipid formed from GDP- $\left[{ }^{14} \mathrm{C}\right]$ mannose and exogenous dolichyl phosphate were found to function as direct mannosyl donors for the synthesis of labelled mannoproteins. These results clearly indicate the existence of dolichol-type glycolipids and their role as intermediates in transglycosylation reactions of this algal system. Both the saturation of the $\alpha$-isoprene unit and the length of the polyprenyl chain may be regarded as evolutionary markers.
\end{abstract}

The molecular basis of complex phenomena such as 'recognition', intercellular adhesion, assembly and autolytic disassembly of highly ordered cell-wall macromolecular lattice structures have been successfully studied in unicellular biflagellate green algae of the Chlamydomonaceae during their vegetative and reproductive cell cycles (Schlösser, 1976; Goodenough, 1977; Catt et al., 1978; Forest et al., 1978; Köhle et al., 1980; Kaska \& Gibor, 1982). A number of observations have clearly indicated that large-molecular-mass glycoproteins play a major part in these developmental processes (Lang \& Chrispeels, 1976; Catt et al., 1976; Lens et al., 1980, 1982; Monk et al., 1983).

The cell wall of these primitive eukaryotes is composed of several concentric layers of glycoproteins (Roberts, 1974; Monk et al., 1983). In many respects they are similar to hydroxyproline-rich

Abbreviations used: Dol- $P$, dolichyl monophosphate; Und- $P$, undecaprenyl monophosphate. cell-wall glycoproteins of higher plants ('extensin'). However, little information is available on the structure and biosynthesis of these macromolecules. In cell-wall preparations from Chlamydomonas gymnogama and Chlamydomonas reinhardii hydroxyproline was found to be $O$-glycosidically linked either to a mixture of hetero-oligosaccharides composed of arabinose and galactose or to monomeric galactose. Other monosaccharides, such as mannose, xylose, glucose or rhamnose, have been detected in total hydrolysates of wall preparations; however, their position within the macromolecules is not known (Miller et al., 1972, 1974; Roberts, 1974; Lang \& Chrispeels, 1976).

Polyprenol-type lipids are known for their involvement as mediators in sugar transfer reactions in the biosynthesis of cell-wall material of bacteria (see, e.g., Mescher, 1981), as well as in the synthesis of glycoproteins and polysaccharides in various eukaryotic cells (Hemming, 1977; Parodi \& Leloir, 1979; Pont Lezica, 1979; Ericson \& 
Elbein, 1980). This is especially well documented for $N$-glycosidic linkages (Hubbard \& Ivatt, 1981). The formation of short manno-oligosaccharides linked through an $O$-glycosidic linkage to peptidyl serine has been studied in yeast. In this case it was established that only the transfer of the initial mannose unit that is directly linked to peptidyl serine was mediated by Dol- $P$, whereas subsequent mannosylation occurred directly from the sugar nucleotide (Babczinski et al., 1980). In more-recent reports from this laboratory (Lang, 1982a,b) it has been shown that a crude membrane preparation from Chlamydomonas reinhardii transferred Dgalactose from UDP-D-galactose to peptidyl hydroxyproline. Since, however, an involvement of lipid intermediates in protein galactosylation has been found to be unlikely in this algal system (Lang, 1982b), it was decided to investigate instead their possible role in mannosyl transfer reactions. The results given in the present paper demonstrate that membranes from Chlamydomonas reinhardii incubated with GDP-D-mannose synthesized mannoproteins via polyprenol mannosyl monophosphate. Exogenously supplied Dol-P and Und$P$ lead to the formation of considerable amounts of their corresponding mannosyl derivatives. The mannolipid formed from endogenous acceptors revealed a chain length similar to that of prokaryotic undecaprenol, but its $\alpha$-isoprene unit was found to be saturated, as in dolichol from eukaryotes.

\section{Experimental}

The strain, materials and methods not explicitly mentioned here were exactly the same as described previously (Lang, 1982a,b).

\section{Chemicals}

GDP-D- $\left[{ }^{14} \mathrm{C}\right]$ mannose $(11.4 \mathrm{GBq} / \mathrm{mmol})$ was obtained from Amersham-Buchler and Dol-P- $\left[{ }^{3} \mathrm{H}\right]-$ mannose $(33.7 \mathrm{GBq} / \mathrm{mmol})$ was from the New England Nuclear Co. Dol-P was from Sigma Chemical Co., and Und- $P$ was generously supplied by Dr. T. Chojnacki, Institute of Biochemistry and Biophysics, Polish Academy of Sciences, Warsaw, Poland. All other chemicals were from E. Merck or from Sigma Chemical Co.

Transfer of mannose residues from $G D P-\left[{ }^{14} C\right]-$ mannose

The standard incubation mixture was usually assayed in a total volume of $50 \mu \mathrm{l}$. It contained $40 \mu \mathrm{l}$ of crude particulate enzyme preparation (Lang, $1982 b), 5 \mu \mathrm{l}$ of $0.1 \mathrm{M}-\mathrm{MgCl}_{2}$ and $5 \mu \mathrm{l}$ of GDP-[14 $\left.\mathrm{C}\right]-$ mannose $(4.6 \mathrm{kBq}$, equal to $405 \mathrm{pmol})$. Unless stated otherwise, all incubations were carried out at $25^{\circ} \mathrm{C}$ for $30 \mathrm{~min}$. When Dol-P $(10 \mu \mathrm{g})$ or Und-P
$(10 \mu \mathrm{g})$ was also present, they were dried under a stream of $\mathrm{N}_{2}$ and solubilized with a final concentration of $0.45 \%(w / w)$ Triton X-100.

Incorporation of radioactivity into lipids was measured by adding $2 \mathrm{ml}$ of chloroform/methanol $(2: 1, \mathrm{v} / \mathrm{v})$ to the assay mixture, followed by a brief centrifugation to separate the insoluble material. After addition of $400 \mu \mathrm{l}$ of $4 \mathrm{mM}-\mathrm{MgCl}_{2}$ and phase separation, the lower phase was washed in accordance with the procedure of Folch et al. (1957). A sample was dried and its radioactivity counted in a xylene-based scintillation cocktail.

Incorporation of radioactivity into the polymer fraction was measured as described by Lang (1982a).

\section{Transfer of mannose residues from Dol-P-[$\left[{ }^{3} \mathrm{H}\right]-$ mannose}

Transfer of $\left[{ }^{3} \mathrm{H}\right]$ mannose was assayed in a total volume of $100 \mu \mathrm{l}$. The incubation mixture was prepared by mixing $0.2 \mathrm{kBq}$ of Dol- $P-\left[{ }^{3} \mathrm{H}\right]$ mannose, $10 \mu \mathrm{l}$ of $0.1 \mathrm{M}-\mathrm{MgCl}_{2}$ and $10 \mu \mathrm{l}$ of $4.5 \%$ (w/w) Triton $\mathrm{X}-100$, and the reaction was started by adding $80 \mu \mathrm{l}$ of crude particulate enzyme preparation. At various times samples were withdrawn and radioactivity in the lipid fraction and in the polymer fraction was determined as described above.

\section{Characterization of mannolipids}

DEAE-cellulose chromatography. 'Folch-washed' mannolipids were placed on small columns $(0.5 \mathrm{~cm} \times 2 \mathrm{~cm})$ of DEAE-cellulose (acetate form) and eluted sequentially with $99 \%$ (v/v) methanol, $0.2 \mathrm{M}$-ammonium acetate in $99 \%(\mathrm{v} / \mathrm{v})$ methanol and $0.4 \mathrm{M}$-ammonium acetate in $99 \%(\mathrm{v} / \mathrm{v})$ methanol. The radioactivity in each eluate was determined.

Mild acid hydrolysis. 'Folch-washed' mannolipids were dried under a stream of $\mathrm{N}_{2}$, redissolved in $1 \mathrm{ml}$ of $10 \mathrm{mM}-\mathrm{HCl}$ in $50 \%(\mathrm{v} / \mathrm{v})$ propan-1-ol (pH 2.0) and incubated at $100^{\circ} \mathrm{C}$. At various times, samples were cooled to room temperature and $0.5 \mathrm{ml}$ of chloroform was added. After a brief centrifugation the two phases were separated. Chloroform was evaporated and radioactivity from both phases was counted in a xylene-based scintillation cocktail.

Phenol treatment. 'Folch-washed' mannolipids were dried and incubated in $50 \%(\mathrm{w} / \mathrm{w})$ phenol at $68-70^{\circ} \mathrm{C}$. At various times, samples were icecooled and both phases were washed and counted for radioactivity as described by García et al. (1974).

\section{Chromatography}

T.1.c. was carried out on silica gel 60 on precoated aluminium foil from Merck. The following solvents were used: $A$, chloroform/propan-2- 
ol/ethanol/1 M-acetic acid $(2: 2: 3: 1$, by vol. $) ; B$, chloroform/methanol/water $(60: 25: 4$, by vol.); $C$, chloroform/methanol/water ( $45: 35: 6$, by vol.).

Molecular-sieve column chromatography was performed by applying 'Folch-washed' mannolipids to a Sephadex G-75 column $(0.9 \mathrm{~cm} \times 73 \mathrm{~cm})$ equilibrated and eluted with $0.5 \%(\mathrm{w} / \mathrm{w})$ sodium deoxycholate in $0.1 \mathrm{M}$-Tris/ $\mathrm{HCl}$ buffer, $\mathrm{pH} 7.4$; $1.4 \mathrm{ml}$ fractions were collected. Blue Dextran was included in all samples in order to mark the void volume of the column.

\section{Results}

Synthesis in vitro of mannolipids and mannoproteins

The synthesis of labelled lipophilic and polymer compounds was performed with membrane preparations from Chlamydomonas reinhardii under conditions similar to those employed with galactosyl transfer studies (Lang, 1982a,b). As shown in Fig. 1, incubation with GDP- $\left[{ }^{14} \mathrm{C}\right]$ mannose resulted in the formation of small amounts of radioactive lipids and polymer products. Both activities required a bivalent cation, with $\mathrm{Mg}^{2+}$ being the most

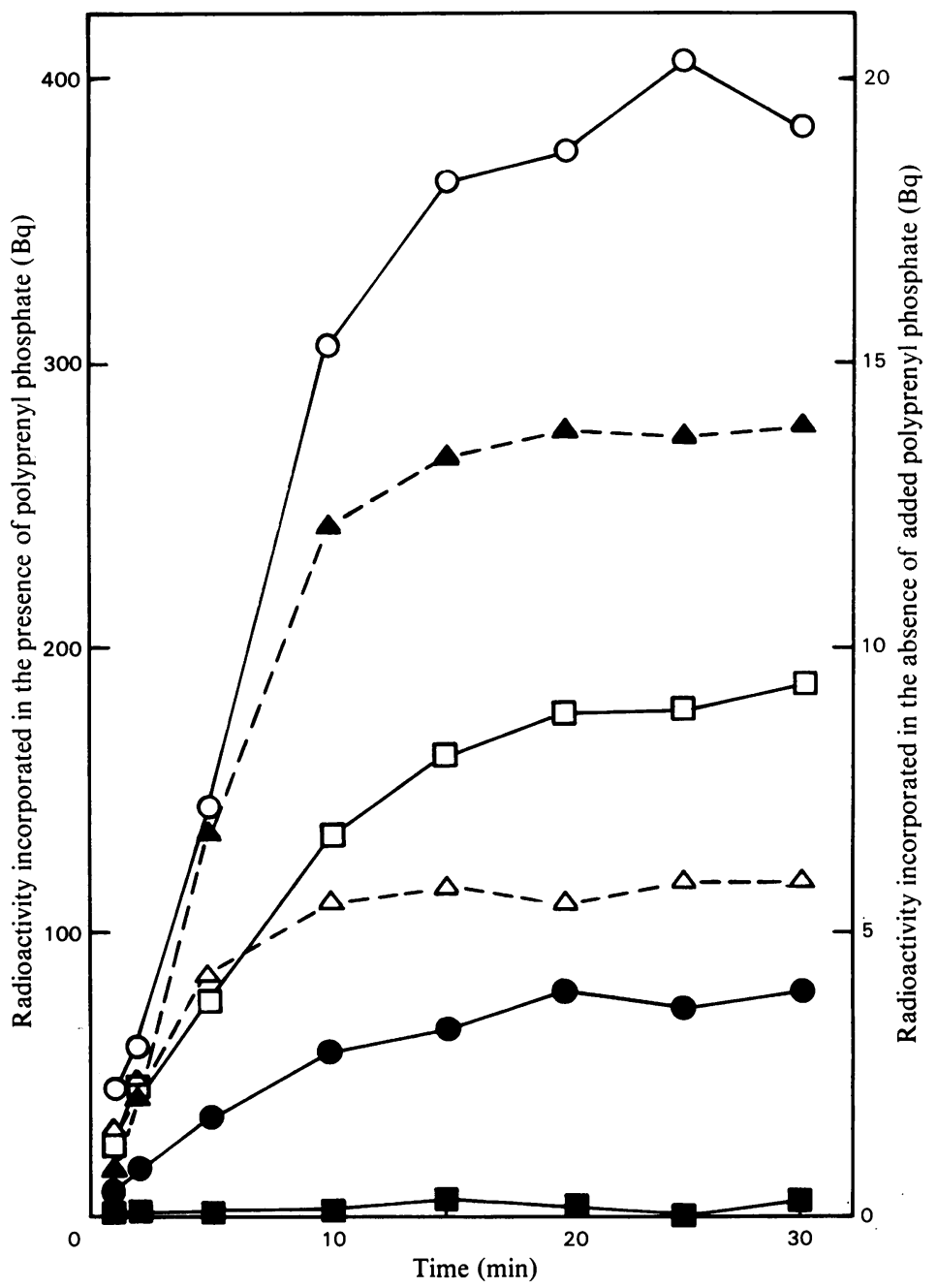

Fig. 1. Time course of incorporation of radioactivity from GDP-D-[ $\left.{ }^{14} C\right]$ mannose into lipids $(\triangle, \bigcirc$ and $\square)$ and polymers $(\Delta, \bigcirc$ and $\square$ ) in the absence ( $\triangle$ and $\Delta$, right-hand scale) and in the presence (left-hand scale) of either $10 \mu \mathrm{g}$ of Dol-P (O and $O)$ or $10 \mu \mathrm{g}$ of Und-P ( $\square$ and $\square)$

The size of the standard incubation mixture was doubled compared with that described in the Experimental section. At various times half of the incubation mixture $(50 \mu \mathrm{l})$ was removed and incorporation of radioactivity into polymers was measured as described by Lang (1982a). A $2 \mathrm{ml}$ volume of chloroform/methanol $(2: 1, \mathrm{v} / \mathrm{v})$ was added to the remaining half, and radioactivity in these lipid fractions was determined after 'Folch-washing' as described in the Experimental section. 


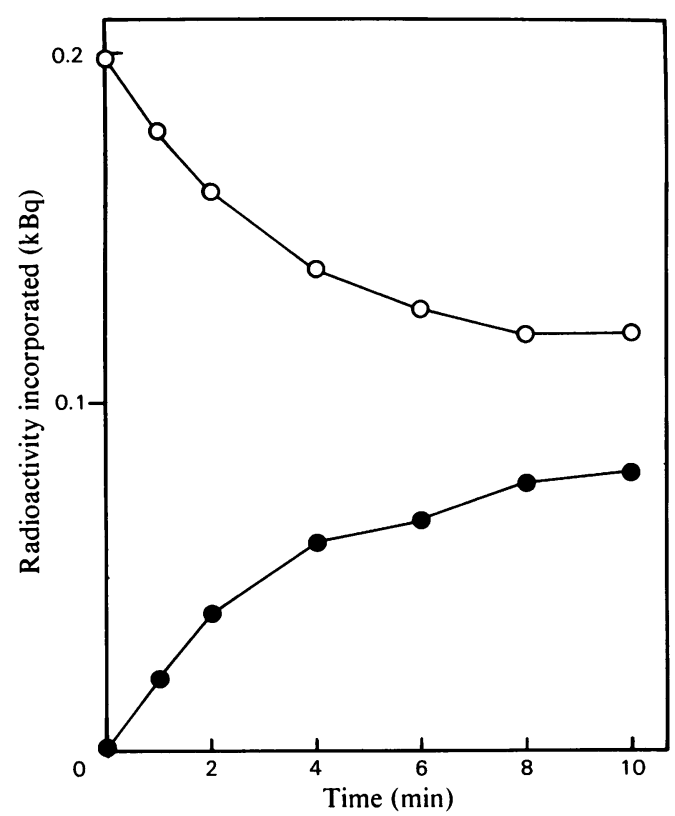

Fig. 2. Time course of direct transfer of mannose from lipid to protein

Crude membranes were incubated with authentic liver Dol-P: $\left[{ }^{3} \mathrm{H}\right]$ mannose in the presence of detergent and $\mathbf{M g}^{2+}$ as described in the Experimental section. $O,\left[{ }^{3} \mathrm{H}\right]$ Mannolipid;,$\left[{ }^{3} \mathrm{H}\right]$ mannoprotein.

effective of the ions tested. Both reactions were found to be inhibited by disodium EDTA (results not shown). The amount of endogenous acceptors present in the membrane preparations was assumed to be a limiting factor, which, among others, determined the amount of products formed. Although low concentrations of detergent were found to inhibit mannolipid formation, the addition of $\alpha$-saturated liver Dol-P or $\alpha$-unsaturated Und-P in the presence of optimum concentrations of Triton X-100 $(0.45 \%, w / w)$ effectively enhanced the amount of radioactive mannolipids formed. Addition of $10 \mu \mathrm{g}$ of exogenous Dol-P resulted in an approximately 70 -fold increase in labelled lipids. This enhancement was accompanied by a 68-fold stimulation of labelled polymer formation. In contrast, the same amount of Und-P stimulated lipid formation about 25-30-fold, whereas the synthesis of labelled polymers was not positively affected (Fig. 1).

\section{Direct transfer of mannose from lipid to protein}

Both authentic Dol- $P$ - $\left[{ }^{3} \mathrm{H}\right]$ mannose and partially purified (t.l.c. in system $B$ ) mannolipid obtained from incubations in vitro of crude membranes with GDP- $\left[{ }^{14} \mathrm{C}\right]$ mannose in the presence of exogenous unlabelled Dol- $P$ were tested directly as

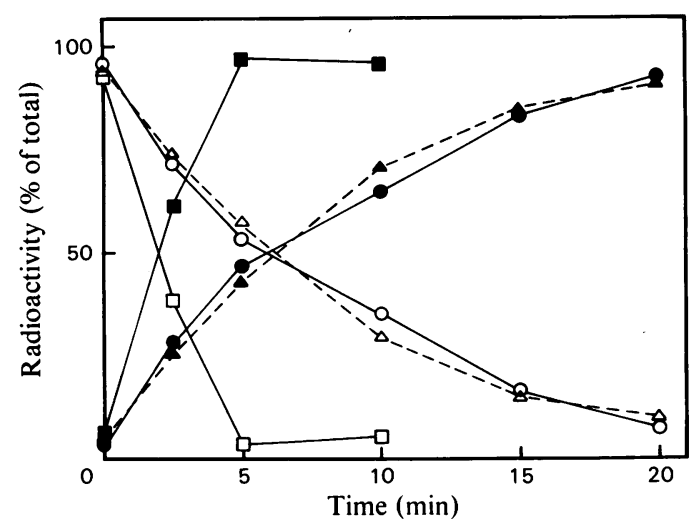

Fig. 3. Time course of mild acid hydrolysis of mannolipids Chloroform/methanol extracts obtained by direct extraction of standard incubation mixtures were treated as described in the Experimental section. At various times samples were rapidly cooled and phases were separated by addition of $0.5 \mathrm{ml}$ of chloroform. Radioactivity was determined in the aqueous phase $(\boldsymbol{Q}, \boldsymbol{\square}$ and $\Delta)$ and in the organic phase $(O, \square$ and $\Delta)$. and $O$, Mannolipid formed in the presence of $10 \mu \mathrm{g}$ of Dol-P; $\square$ and $\square$, mannolipid formed in the presence of $10 \mu \mathrm{g}$ of Und-P; $\triangle$ and $\Delta$, endogenous mannolipid formed without further additions.

mannosyl donors for mannoprotein synthesis. As shown in Fig. 2, labelled mannose is released from Dol- $P-\left[{ }^{3} \mathrm{H}\right]$ mannose in a time-dependent reaction and concomitantly is transferred to the polymer fraction. Similar results were obtained with the mannolipid from GDP- $\left[{ }^{14} \mathrm{C}\right]$ mannose and Dol-P(results not shown).

\section{Properties of mannolipids}

Since extraction with chloroform/methanol of the incubation mixtures yielded a single radioactive compound (Figs. 5 and 6), it was possible to characterize each compound without further purification. After mild acid treatment of the lipid fraction obtained from endogenous and exogenous acceptors, the only radioactive product detected in the aqueous phase after phase separation was Dmannose (results not shown). Thus no epimerization of the mannose from GDP- $\left[{ }^{14} \mathrm{C}\right]$ manose occurred in the assay mixtures.

Mild acid treatment degraded the mannolipids from the endogeneous acceptor as well as from exogenous liver Dol- $P$ and from Und- $P$. The time course of the degradation of the endogenous acceptor lipid followed the characteristics of $\alpha$-saturated liver Dol- $P$-mannose and was clearly distinguishable from that of Und-P-mannose, which, according to its $\alpha$-unsaturated structure, is cleaved more rapidly (Fig. 3). The results also indicate that $C-1$ 


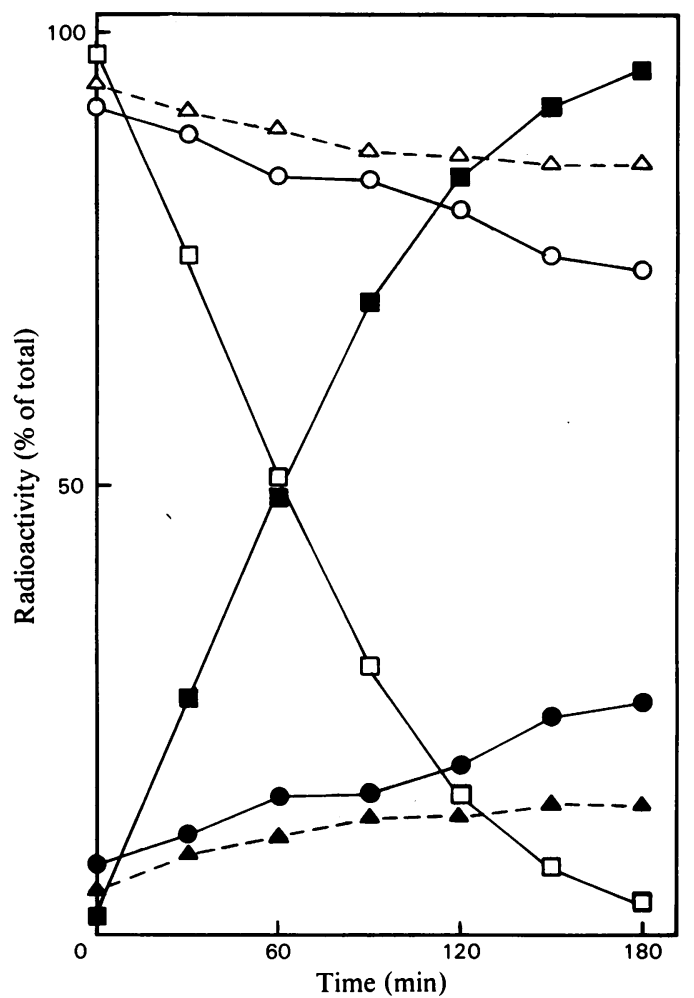

Fig. 4. Time course of hot-phenol hydrolysis of mannolipids Chloroform/methanol extracts were treated as described in the Experimental section. At various times samples were ice-cooled for phase separation. Radioactivity was determined in the aqueous phase $(O, \square$ and $\Delta)$ and in the phenolic phase $(O, \square$ and $\triangle)$. and $O$, Mannolipid formed in the presence of $10 \mu \mathrm{g}$ of Dol-P; $\square$ and $\square$, mannolipid formed in the presence of $10 \mu \mathrm{g}$ of Und- $P ; \Delta$ and $\Delta$, endogenous mannolipid formed without further additions.

of the mannose is glycosidically bound to the phosphate group because a linkage to any other hydroxy group of the mannose molecule would not be hydrolysed by such a mild procedure.

The presence of a saturated isoprenyl unit linked to the phosphate group in the endogenous acceptor lipid has been confirmed by results obtained from hot-phenol treatment (Fig. 4). The resistance to this treatment was found to be similar to that of Dol-P-Man, and was clearly different from Und-PMan, which was completely cleaved after $180 \mathrm{~min}$ under the conditions used.

Binding to DEAE-cellulose and elution with increasing concentrations of ammonium formate or ammonium acetate has been generally used to demonstrate the existence of monophosphate and diphosphate groups in polyprenyl-type glycolipids. The endogenous Chlamydomonas mannolipid, as well as the mannolipids obtained from exogenous Dol- $P$ and Und- $P$, were found to bind to the resin in the presence of $99 \%$ methanol, but were eluted with $0.2 \mathrm{M}$-ammonium acetate in $99 \%$ methanol (Table 1). These results demonstrate the acidic nature of the lipids and also clearly indicate the sole existence of monophosphate groups.

Several t.l.c. separation systems have been employed in these studies in order to identify the mannolipids and to estimate tentatively their molecular size. Solvent $C$ on silica gel separates polyprenol monophosphate monosaccharides from retinyl monophosphate monosaccharides and from polyprenol diphosphate derivatives (Creek et al., 1983). In this system the endogenous mannolipids and both lipids from the exogenous acceptors cochromatographed with an authentic sample of Dol- $P-\left[{ }^{3} \mathrm{H}\right]$ mannose. Spots at $R_{F}$ values expected for retinyl derivatives and lipid diphosphate sugars were not detected (results not shown).

Solvents $A$ (Quesada-Allue \& Parodi, 1983) and $B$ (Pont Lezica et al., 1975) are known to separate polyprenyl monophosphate monosaccharides according to their chain length. In both systems the mannolipid formed in the presence of exogenous Dol- $P$ migrated the same distance as a long-chain $\left(\mathrm{C}_{85}-\mathrm{C}_{105}\right)$ Dol- $P-\left[{ }^{3} \mathrm{H}\right]$ mannose standard. In contrast, the endogenous mannolipid ran a shorter distance, in approximately the same position as the mannolipid formed from exogenous Und-P (Fig. 5).

The molecular size of the isoprenoid glycolipids was also examined by gel filtration on Sephadex G-

Table 1. Fractionation on DEAE-cellulose of mannolipids synthesized in the presence and in the absence of exogenous Dol-P or Und-P

Each sample was applied to the resin in $99 \%(\mathrm{v} / \mathrm{v})$ methanol followed by stepwise elution with $(a) 99 \%$ methanol, $(b)$ $0.2 \mathrm{M}$-ammonium acetate in $99 \%$ methanol and (c) $0.4 \mathrm{M}$-ammonium acetate in $99 \%$ methanol. Data are given in Bq/ total fraction after subtraction of background radioactivity. For further details see the Experimental section.

Radioactivity (Bq/fraction)

$\begin{array}{llllll}\text { Assay conditions } & \text { Fraction } & \ldots & (a) & (b) & (c) \\ \text { Dol- } P \text { added } & & & 2.0 & 86.6 & 1.3 \\ \text { Und- } P \text { added } & & & 2.5 & 45.0 & 2.5 \\ \text { No additions } & & & 1.9 & 17.8 & 1.9\end{array}$




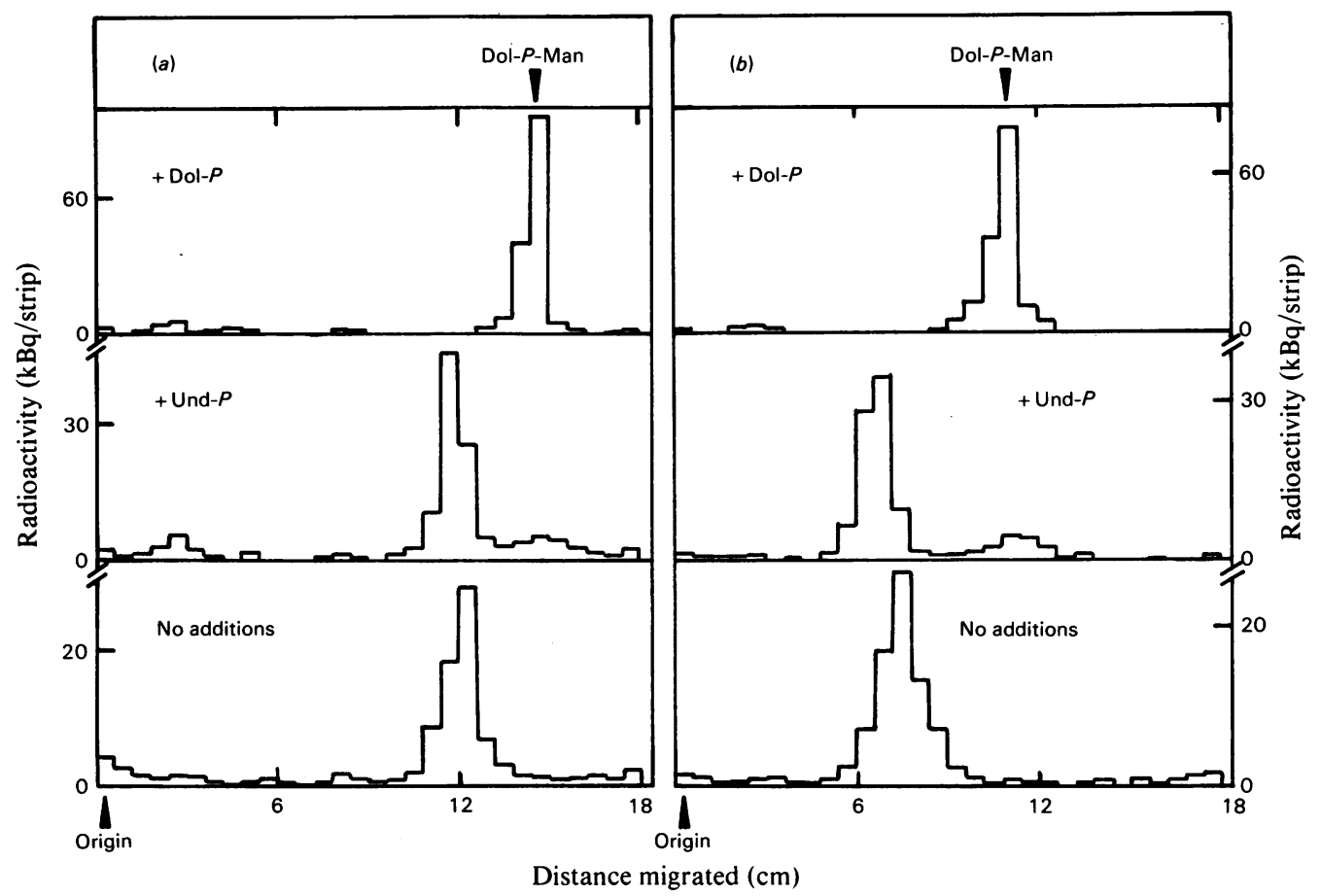

Fig. 5. Analyses of mannolipids by t.l.c.

Mannolipids obtained in the absence and in the presence of exogenous acceptors (10 $\mu \mathrm{g}$ of Dol-P or $10 \mu \mathrm{g}$ of Und-P) were applied to precoated $20 \mathrm{~cm} \times 20 \mathrm{~cm}$ silica gel G 60 thin layers and separately run in solvent $A(a)$ and solvent $B$ (b) in the presence of a Dol- $P-\left[{ }^{3} \mathrm{H}\right]$ mannose standard whose $R_{F}$ value in each system is indicated by the arrow in the uppermost sections. The thin layer on aluminium foil was dried, the foil was cut into small strips $(0.6 \mathrm{~cm})$ and the radioactivity of each strip was counted in a toluene-based scintillation cocktail.

75 in a buffered solution of sodium deoxycholate. The detergent forms inclusion compounds with the lipids in question, and the number of deoxycholate molecules involved is thought to depend on the chain length of the lipid (Quesada-Allue \& Parodi, 1983). Comparison with a Dol- $P-\left[{ }^{3} \mathrm{H}\right]$ mannose standard showed again that the mannosylated endogenous lipid formed in the absence of exogenous acceptors has about the same size as Und$\boldsymbol{P}$-mannose and can be clearly separated from the lipid formed from Dol- $P$, which co-eluted with the standard. A sample of labelled monogalactosyl diacyl glyceride, which is synthesized in this system in vitro when UDP- $\left[{ }^{14} \mathrm{C}\right]$ galactose is supplied (Lang, 1982b), was also found to be clearly separable from the isoprenoid mannolipids in this separation system (Fig. 6).

\section{Discussion}

These results demonstrate that a crude membrane preparation from Chlamydomonas reinhardii incubated with GDP-D- $\left[{ }^{14} \mathrm{C}\right]$ mannose in the presence of $\mathrm{Mg}^{2+}$ synthesizes a $\left[{ }^{14} \mathrm{C}\right]$ mannolipid that has the properties of an $\alpha$-saturated polyprenyl mannophosphate and a chain length similar to or slightly longer than (Fig. 4) bacterial undecaprenol $\left(\mathrm{C}_{55}\right)$. In the same incubation mixture $\left[{ }^{14} \mathrm{C}\right]-$ mannose was also incorporated into a trichloroacetic acid-insoluble polymer fraction, which most probably represents mannoproteins. Exogenous long-chain Dol- $P\left(\mathrm{C}_{85}-\mathrm{C}_{105}\right)$ very effectively stimulated incorporation of radioactivity from GDP- $\left[{ }^{14} \mathrm{C}\right]$ mannose into the lipid fraction (Fig. 1). The labelled compound formed was found to be identical with commercially available long-chain Dol- $P-\left[{ }^{3} \mathrm{H}\right]$ mannose. The tremendous increase (about 70-fold) in mannolipid formation with exogenous Dol- $P$ in the presence of optimum concentrations of Triton X-100 $(0.45 \%, w / w)$ was accompanied by a 7-fold increase in the incorporation rate into mannoproteins. This result indicates that dolichol-type mannolipid formed from GDPmannose is an intermediate in protein mannosylation. Additional evidence for this hypothesis is provided by the experiments in which both authentic liver Dol-P-[ $\left[{ }^{3} \mathrm{H}\right]$ mannose (Fig. 2) and partially purified $\left[{ }^{14} \mathrm{C}\right]$ mannolipid formed from GDP- 


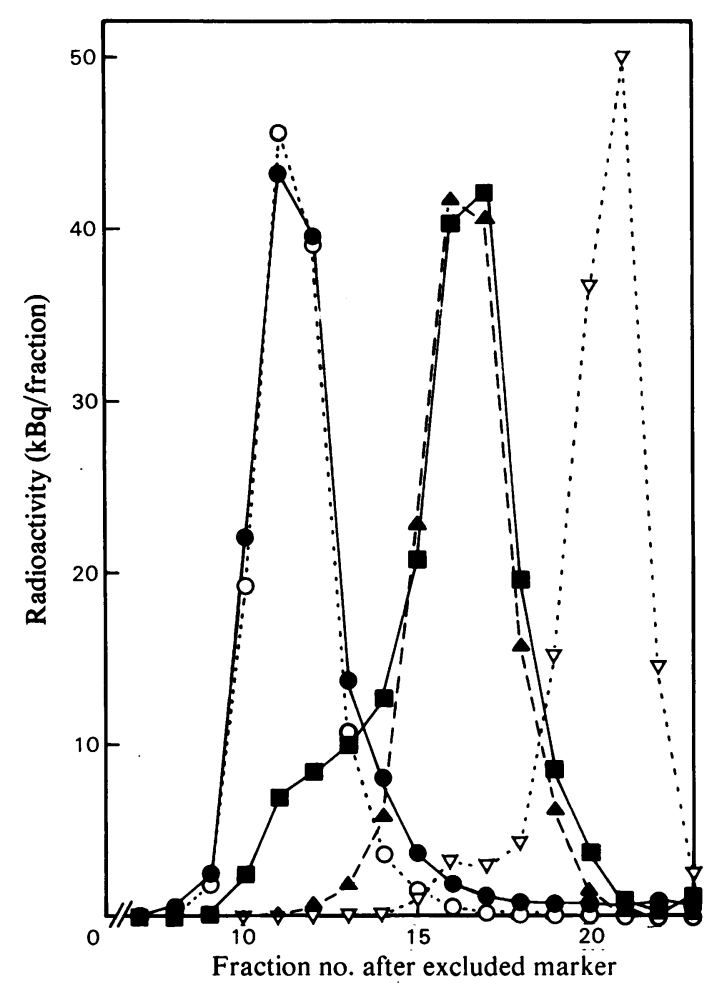

Fig. 6. Analyses of mannolipids by gel filtration Mannolipids with about equal radioactive labelling, obtained in the absence $(\boldsymbol{A})$ and in the presence of exogenous acceptors $(10 \mu \mathrm{g}$ of Dol-P, $; 10 \mu \mathrm{g}$ of Und- $P, \square)$, were successively passed through a column $(0.9 \mathrm{~cm} \times 73 \mathrm{~cm})$ of Sephadex G-75 in $0.1 \mathrm{M}$ Tris $/ \mathrm{HCl}$ buffer, $\mathrm{pH} 7.4$, containing $0.5 \%$ sodium deoxycholate. For calibration a long-chain Dol- $P$ $\left[{ }^{3} \mathrm{H}\right]$ mannose standard $(\mathrm{O})$ and a sample of $\left[{ }^{14} \mathrm{C}\right]-$ galactose-labelled monogalactosyl diacyl glyceride $(\nabla)$ was also passed through the column. Dextran Blue was included in all runs for determination of the exclusion volume. Fractions of volume $1.4 \mathrm{ml}$ were collected and their radioactivity was determined after mixing with a xylene-based scintillation cocktail containing Triton $\mathrm{X}-100$.

$\left[{ }^{14} \mathrm{C}\right]$ mannose and Dol- $P$ were shown to serve as substrates for protein mannosylation in a timedependent reaction. It also rules out the possibility that the effect of addition of Dol- $P$ is solely due to its detergent property. Und- $P$, a short-chain $\left(\mathrm{C}_{55}\right)$ isoprenoid, which in contrast with dolichyl derivatives is unsaturated in its $\alpha$-isoprene unit, was also accepted as exogenous substrate to yield a compound with the properties of Und- $P-\left[{ }^{14} \mathrm{C}\right]-$ mannose. Compared with Dol-P stimulation, the rates of Und- $P$-mannose synthesis were about onethird under conditions in which the molar concentration of Und-P was about twice as high as the concentration of Dol- $P$. In contrast with Dol- $P$, however, the mannose moiety from Und- $P-\left[{ }^{14} \mathrm{C}\right]-$ mannose was not further transferred to the polymer fraction (Fig. 1). Apparently, the presence of an $\alpha$-saturated isoprene unit and not the chain length is the essential feature for a lipid to act as an intermediate in protein mannosylation in this eukaryotic system.

The mannolipid formed in the absence of exogenous acceptors reveals the presence of a monophosphate group, as evidenced by its behaviour on DEAE-cellulose (Table 1). The time course of the hydrolytic cleavage of the mannose residue (Fig. 3) has been found to be similar to that of Dol-Pmannose and was clearly distinguishable from that of Und-P-mannose, which, in accordance with its polyallylic nature, is hydrolysed faster (QuesadaAllue \& Parodi, 1983). The presence of an $\alpha$-saturated isoprene unit is also clearly indicated by its stability to hot-phenol treatment, which was found to be similar to that of Dol-P-mannose (Fig. 4).

The chain length of the carbon skeleton reported for dolichol-type glycolipids from a number of different eukaryotic systems is in the range $\mathrm{C}_{85^{-}}$ $\mathrm{C}_{105}$. The endogenous product formed in these studies was found to chromatograph on silica gel $G$ in solvent $C$ with an $R_{F}$ value of 1.0 , which is characteristic for all polyprenol monophosphate monosaccharides (Creek et al., 1983). However, it was separated both from long-chain Dol- $P-\left[{ }^{3} \mathrm{H}\right]-$ mannose standard and from long-chain Dol-P$\left[{ }^{14} \mathrm{C}\right]$ mannose formed from exogenously supplied Dol- $P$ in solvents $A$ and $B$, indicating that a product with a shorter carbon chain is the endogenous acceptor in these membrane preparations. Cochromatography with Und- $P-\left[{ }^{14} \mathrm{C}\right]$ mannose synthesized from exogenous Und- $P$ in both of these solvents indicates that its chain length may be either similar to or slightly longer than $\mathrm{C}_{55}$ (Fig. 5). The elution pattern observed in molecular-sieve column chromatography studies with Sephadex G-75 in the presence of buffered deoxycholate provides additional evidence for this conclusion (Fig. 6).

The involvement of lipid intermediates in glycosyl transfer pathways is ubiquitous in both prokaryotic and eukaryotic organisms. Until recently both the chain length and the saturated structure of the $\alpha$-isoprene unit were generally regarded as principal differences in eukaryotes and prokaryotes. However, there are now some published results indicating that some exceptions must be taken into account: (a) $\alpha$-saturated short-chain lipids have been reported in pig liver (Radominska-Pyrek et al., 1979) and in the trypanosomatid Crithidia fasciculata (Quesada-Allue \& Parodi, 1983), (b) a prokaryote-like unsaturated $\mathrm{C}_{55}$ compound was found in bovine pituitary (Mańkowski 
et al., 1976), and (c) an unsaturated long-chain lipid has been described in hen oviduct (Hayes \& Lucas, 1980). However, evidence for the involvement of these lipids in sugar transfer has not always been presented.

The results reported in the present paper may be regarded as a further exception to this rule, because they demonstrate another short-chain polyprenoltype lipid intermediate involved in glycoprotein biosynthesis in an eukaryotic organism. On the other hand, they confirm the concept that there is a tendency in evolution towards the enlargement of the carbon skeleton of polyprenols involved in glycosyl transfer reactions. The saturated shortchain lipids from primitive eukaryotes such as Crithidia fasciculata and Chlamydomonas reinhardii may be interpreted as 'missing links' between prokaryotes and eukaryotes, exhibiting conservative short-chain length but progressive saturation of the $\alpha$-isoprene unit.

The results presented provide a clear indication that Chlamydomonas reinhardii can synthesize a mannosyl phosphoryl polyprenol that is involved in mannoprotein biosynthesis. The identity of the labelled protein remains to be determined.

\section{References}

Babczinski, P., Haselbeck, A. \& Tanner, W. (1980) Eur. J. Biochem. 105, 509-515

Catt, J. W., Hills, G. J. \& Roberts, K. (1976) Planta 131, $165-171$

Catt, J. W., Hills, G. J. \& Roberts, K. (1978) Planta 138, 91-98

Creek, K. E., Morré, D. J., Silverman-Jones, C. S., Shidoji, Y. \& De Luca, L. M. (1983) Biochem. J. 210, 541-547

Ericson, M. C. \& Elbein, A. D. (1980) in The Biochemistry of Plants (Preiss, J., ed.), vol. 3, pp. 589-616, Academic Press, London and New York

Folch, J., Lees, M. \& Sloane-Stanley, G. H. (1957) J. Biol. Chem. 226, 497-509
Forest, C. L., Goodenough, D. A. \& Goodenough, U. W. (1978) J. Cell Biol. 79, 74-84

García, R. C., Recondo, E. \& Dankert, M. (1974) Eur. J. Biochem. 43, 93-105

Goodenough, U. W. (1977) in Microbial Interactions (Reissig, J. L., ed.), pp. 325-350, Chapman and Hall, London

Hayes, G. R. \& Lucas, J. J. (1980) J. Biol. Chem. 255, 7536-7539

Hemming, F. W. (1977) Biochem. Soc. Trans. 5, 12231231

Hubbard, S. C. \& Ivatt, R. J. (1981) Annu. Rev. Biochem. 50, 553-583

Kaska, D. D. \& Gibor, A. (1982) Exp. Cell Res. 138, 121125

Köhle, D., Lang, W. \& Kauss, H. (1980) Arch. Microbiol. 127, 239-243

Lang, W. C. (1982a) Plant Physiol. 69, 678-681

Lang, W. C. (1982b) Plant Cell Physiol. 23, 1259-1266

Lang, W. C. \& Chrispeels, M. J. (1976) Planta 129, 183189

Lens, P. F., van den Briel, W., Musgrave, A. \& van den Ende, H. (1980) Arch. Microbiol. 126, 77-81

Lens, P. F., Olofsen, F., Nederbragt, A., Musgrave, A. \& van den Ende, H. (1982) Arch. Microbiol. 131, 241-246

Mańkowski, T., Jankowski, W., Chojnacki, R. \& Franke, P. (1976) Biochemistry 15, 2125-2130

Mescher, M. F. (1981) Trends Biochem. Sci. 6, 97-99

Miller, D. H., Lamport, D. T. \& Miller, M. (1972) Science 176, 918-920

Miller, D. H., Mellman, I. S., Lamport, D. T. \& Miller, M. (1974) J. Cell Biol. 63, 420-429

Monk, B. C., Adair, W. S., Cohen, R. A. \& Goodenough, U. W. (1983) Planta 158, 517-533

Parodi, A. J. \& Leloir, L. F. (1979) Biochim. Biophys. Acta 559, 1-37

Pont Lezica, R. (1979) Biochem. Soc. Trans. 7, 334-337

Pont Lezica, R., Brett, C. T., Martinez, P. R. \& Dankert, M. A. (1975) Biochem. Biophys. Res. Commun. 66, 980987

Quesada-Allue, L. A. \& Parodi, A. J. (1983) Biochem. J. 212, 123-128

Radominska-Pyrek, A., Chojnacki, T. \& Pyrek, S. (1979) Biochem. Biophys. Res. Commun. 86, 395-401

Roberts, K. (1974) Philos. Trans. R. Soc. London Ser. B 268, 129-146

Schlösser, U. G. (1976) Ber. Dtsch. Bot. Ges. 89, 1-56 